\title{
BANACH LATTICES WITH THE SUBSEQUENCE SPLITTING PROPERTY
}

\author{
LUTZ W. WEIS
}

(Communicated by William J. Davis)

\begin{abstract}
A Banach lattice $X$ has SSP if every bounded sequence in $X$ has a subsequence that splits into a $X$-equi-integrable sequence and a sequence with pairwise disjoint support. We characterize such lattices in terms of uniform order continuity conditions and ultrapowers. This implies that rearrangement invariant function spaces with the Fatou-property have SSP.
\end{abstract}

\section{INTRODUCTION}

In [11] Kadec and A. Pełczyński made the observation that every bounded sequence $\left(f_{n}\right)$ of $L_{p}[0,1], 1 \leq p<\infty$, has a subsequence that splits into two 'extreme' sequences $\left(g_{k}\right)$ and $\left(h_{k}\right)$, where the $h_{k}$ 's have pairwise disjoint support and the $g_{k}$ 's are $L_{p}$-equi-integrable, i.e. $\sup _{k}\left\|\chi_{A} g_{k}\right\|_{L_{p}} \rightarrow 0$ for $\mu(A) \rightarrow 0$.

They used this fact to study the subspace structure of $L_{1}[0,1]$. Soon, this idea proved to be useful also in various other contexts and it was observed that the above splitting is possible in more general Banach function spaces. E.g. in [4 and 10] such splittings appear in the study of isomorphic embeddings of Banach function spaces and it was pointed out that they are possible in Orlicz function spaces with the $\Delta_{2}$-condition and in $q$-concave lattices (cf. [7]). Equiintegrable and pairwise disjoint sequences play an important part in the study of compactness properties of positive operators (see e.g. [6, 7, 15 and 13]) and, more recently, such splittings of sequences were considered in the context of ergodic theorems for positive contractions in [1]. It follows from this work, that the above splitting is also possible in Banach lattices with uniformly monotone norm.

On the other hand, there are Banach lattices with sequences for which the splitting is not possible. A simple example is $c_{0}$, but Figiel, Ghoussoub and Johnson also have constructed reflexive, $p$-convex Banach lattices without the subsequence splitting property. This raises the question: what kind of structural

Received by the editors February 27, 1988.

1980 Mathematics Subject Classification (1985 Revision). Primary 46B25, 46B30.

Key words and phrases. Equi-integrable sets, ultrapowers, rearrangement invariant function spaces. 
property of Banach lattices makes the splitting work? In this paper we give two characterizations of the subsequence splitting property. One of them in terms of a 'uniform' order continuity of $X$ (Theorem 2.8), the second in terms of ultrapowers $X_{\mathfrak{a}}$ of $X$ (Theorem 2.5). While the sufficient conditions we quoted in the first paragraph require the whole ultrapower $X_{\mathfrak{A}}$ to have order continuous norm, we show that order-continuity of a relatively 'small' band $\tilde{X}$ of $X_{\mathfrak{a}}$ is already necessary and sufficient. In a sense made precise in $\S 1, \widetilde{X}$ is the 'same' function space as $X$ on a 'larger' measure space.

We also find a large new class of spaces with the subsequence splitting property, namely all rearrangement invariant function spaces with the Fatouproperty (equivalently, with no subspace isomorphic to $c_{0}$, see Corollary 2.6).

\section{Preliminaries on Ultraproducts and equi-INTEgRable Sets}

In this paper $X$ is always a Banach lattice with order continuous norm, which has a weak unit. By a general representation theorem (see e.g. $[12,1.6 .14]$ ) $X$ can be represented as a lattice ideal of $L_{1}(\Omega, \Sigma, \mu)$ on some probability space $(\Omega, \Sigma, \mu)$ such that

$$
L_{\infty}(\Omega, \Sigma, \mu) \subset X \subset L_{1}(\Omega, \Sigma, \mu) .
$$

In the following we always assume that $X$ is given in this way. Let $\mathfrak{A}$ be a free ultrafilter on $\mathbf{N}$. The ultraproduct $X_{\mathfrak{a}}$ of $X$ is defined as the quotient

$$
X_{\mathfrak{A}}=l_{\infty}(X) / M, \quad M=\left\{\left(f_{n}\right) \in l_{\infty}(X): \mathfrak{A}-\lim \left\|f_{n}\right\|=0\right\} .
$$

$f=\left[f_{n}\right]$ denotes the equivalence class of the sequence $\left(f_{n}\right) \subset X$ and $\|f\|=$ $\mathfrak{A}-\lim \left\|f_{n}\right\|_{X} \cdot X_{\mathfrak{A}}$ is also a Banach lattice with $\left[f_{n}\right] \wedge\left[g_{n}\right]=\left[f_{n} \wedge g_{n}\right]$. (See [9] for details of this construction.)

1.1. Definition. By $\tilde{X}$ we denote the band $\{1\}^{\perp \perp}$ in $X$ generated by $\left[f_{n}\right]$, $f_{n} \equiv 1$, and $\tilde{X}^{\perp}$ is the orthogonal band of $\tilde{X}$ in $X_{\mathfrak{A}}$.

Following a construction in [3] we can represent $\tilde{X}$ as a Banach function lattice: For $L=L_{1}(\Omega, \Sigma, \mu), \tilde{L}$ is an abstract $L$-space which can be represented as $L_{1}(\widetilde{\Omega}, \widetilde{\Sigma}, \widetilde{\mu})$ in such a way that the elements $\left[\chi_{A_{n}}\right] \in \widetilde{L}, A_{n} \in \Sigma$, correspond to characteristic functions $\chi_{A}, A \in \widetilde{\Sigma}$, with $\tilde{\mu}(A)=\left\|\left[\chi_{A_{n}}\right]\right\|_{L}=\mathfrak{A}-\lim \mu\left(A_{n}\right)$. Now the lattice embeddings in (1) extend to lattice embeddings

$$
L_{\infty}(\tilde{X}, \widetilde{\Sigma}, \tilde{\mu}) \subset \widetilde{X} \subset L_{1}(\widetilde{\Omega}, \widetilde{\Sigma}, \tilde{\mu})
$$

1.2. Examples. (a) If $X$ is a purely atomic lattice then $\tilde{X}=X$.

(b) A rearrangement invariant function space $X$ on $(\Omega, \Sigma, \mu)$ is of the form

$$
X=\left\{f \in L_{1}(\Omega, \mu): f^{*} \in X_{0}\right\}, \quad\|f\|_{X}=\left\|f^{*}\right\|_{X_{0}}
$$

where $f^{*}$ is the decreasing rearrangement of $f$ on $[0,1]$ and $X_{0}$ is a rearrangement invariant function space on $[0,1]$ (see $[2,18.2])$. Then

$$
\tilde{X}=\left\{f \in L_{1}(\widetilde{\Omega}, \tilde{\mu}): f^{*} \in X_{0}\right\}, \quad\|f\|_{\widetilde{X}}=\left\|f^{*}\right\|_{X_{0}} .
$$


In particular, for $1 \leq q<\infty: \overparen{L_{q}(\Omega, \mu)}=L_{q}(\widetilde{\Omega}, \tilde{\mu})$.

Proof. (a) Choose $\Omega_{m} \subset \Omega$ such that $\Omega \backslash \Omega_{m}$ consists of finitely many atoms and $\mu\left(\Omega_{m}\right) \rightarrow 0$. If $0 \leq\left[f_{n}\right] \in \widetilde{X}$ then $\beta_{m}=\mathfrak{A}-\lim _{n}\left\|\left.f_{n}\right|_{\Omega_{m}}\right\| \rightarrow 0$ for $m \rightarrow \infty$. Otherwise there are $\varepsilon>0$ and $U_{m} \in \mathfrak{A}$ with $U_{m} \supset U_{m+1} \supset \cdots, \cap U_{m}=\varnothing$, such that $\left\|\left.f_{n}\right|_{\Omega_{m}}\right\| \geq \varepsilon$ for $n \in U_{m}$. Then $g=\left[g_{n}\right]$ with $g_{n}=\left.f_{n}\right|_{\Omega_{m}}$ for $n \in U_{m} \backslash U_{m+1}$ satisfies $0 \leq g \leq f$ and $g \in \widehat{X}^{\perp}$ which contradicts $\|g\| \geq \varepsilon$. $\beta_{m} \rightarrow 0$ implies that $f=\mathfrak{A}-\lim f_{n}$ exists in the norm of $X$ and $\left[f_{n}\right]=[f]$.

(b) Let $0 \leq f \in \widetilde{X}$. By Proposition 1.5 below there is a sequence $f_{n} \in X$ with $f=\left[f_{n}\right]$ which is equi-integrable in $L_{1}(\Omega, \mu)$. Denote by $D(t)$ and $D_{n}(t)$ the distribution functions of $f \in \tilde{X}$ and $f_{n} \in X$, i.e. $D(t)=\tilde{\mu}(|f|>t)$, $D_{n}(f)=\mu\left(\left|f_{n}\right|>t\right)$. The functions $D_{n}, n \in \mathbf{N}$, are equi-continuous on $[0, \infty)$ and $\mathfrak{A}-\lim _{n} D_{n}(t)=D(t)$ for all $t \in[0, \infty)$ by [3, Proposition 1.3]. Hence $D=\mathfrak{A}-\lim D_{n}$ in the sup-norm and

$$
\|f\|_{\widetilde{X}}=\mathfrak{A}-\lim _{n}\left\|f_{n}\right\|_{X}=\mathfrak{A}-\lim \left\|f_{n}^{*}\right\|_{X_{0}}=\left\|f^{*}\right\|_{X_{0}} .
$$

1.3. Proposition. For all $f \in \tilde{X}$ we have $\|f\|=\sup _{m}\|f \wedge m 1\|$.

Proof. Let $f=\left[f_{n}\right] \in \tilde{X}$ with $\left\|f_{n}\right\|=1$. For $\varepsilon>0$ choose $c_{n}$ with $\| f_{n} \wedge$ $c_{n} 1 \|=1-\varepsilon$. We have to show that there is an $m$ with $U_{m}=\left\{n: c_{n} \leq m\right\} \in \mathfrak{A}$ because then

$$
\left\|f_{n} \wedge m 1\right\| \geq\left\|f_{n} \wedge c_{n} 1\right\|=1-\varepsilon \text { for all } n \in U_{m}
$$

and the claim follows. Otherwise we have $\mathfrak{A}-\lim c_{n}=\infty$ and $g_{n}=f_{n}-f_{n} \wedge$ $c_{n} 1 \rightarrow 0$ in measure. Then $g=\left[g_{n}\right] \in \widetilde{X}^{\perp}$ but also $g \leq f \in \widetilde{X}$. This implies $\mathfrak{A}-\lim \left\|g_{n}\right\|=0$ which contradicts $\left\|g_{n}\right\| \geq \varepsilon$.

1.4. Remark. A subset $M \subset X$ is called equi-integrable in $X$ if

$$
\sup \left\{\left\|\chi_{A} f\right\|: f \in M, \mu(A) \leq \delta\right\} \rightarrow 0 \text { for } \delta \rightarrow 0 .
$$

It follows from the order continuity of $X$ that $M$ is equi-integrable in $X$ iff $\sup \{\||f|-|f| \wedge t 1\|: f \in M\} \rightarrow 0$ for $t \rightarrow \infty$.

1.5. Proposition. Let $f=\left[f_{n}\right] \in X_{\mathfrak{A}}$.

(a) $f$ belongs to $\widetilde{X}^{\perp}$ if and only if $\mathfrak{A}-\lim f_{n}=0$ with respect to the topology of convergence in measure,

(b) $f$ is an order continuous element of $X$ (i.e. $\left\|\chi_{A} f\right\|_{\widetilde{X}} \rightarrow 0$ for $\tilde{\mu}(A) \rightarrow 0$ ) if and only if $f$ has a representation $f=\left[f_{n}\right]$ with an equi-integrable sequence in $X$.

Proof. (a) Since $\widetilde{X}^{\perp}=[1]^{\perp}$ we have that $\left[f_{n}\right] \in \widetilde{X}^{\perp}$ iff

$$
\mathfrak{A}-\lim _{n}\left\|\left|f_{n}\right| \wedge m 1\right\|=0 \text { for all } m \text {. }
$$

(b) If the sequence $\left(f_{n}\right)$ is equi-integrable in $X$ then

$$
\left\|\chi_{A} f\right\|_{\tilde{X}} \leq \sup _{n}\left\{\left\|\chi_{B} f_{n}\right\|: \mu(B) \leq \tilde{\mu}(A)\right\} \rightarrow 0 \text { for } \tilde{\mu}(A) \rightarrow 0 .
$$


Now let $0 \leq f=\left[f_{n}\right] \in \tilde{X}$ be an order continuous element. Choose a sequence $\left(n_{m}\right)$ such that

$$
\left\|f-f \wedge n_{m} 1\right\|_{\widetilde{X}} \leq 2^{-m}
$$

Put $g^{1}=f \wedge n_{1} 1$ and choose $g_{n}^{1} \leq n_{1} 1$ with $g^{1}=\left[g_{n}^{1}\right]$. For each $m>1$ choose a sequence $\left(g_{n}^{m}\right)_{n \in \mathbf{N}}$ with

$$
\begin{gathered}
g^{m}=\left[g_{n}^{m}\right]=f \wedge n_{m+1} 1-f \wedge n_{m} 1, \quad g_{n}^{m} \leq\left(n_{m+1}-n_{m}\right) 1 \\
\left\|g_{n}^{m}\right\|_{X}=\left\|g^{m}\right\|_{\widetilde{X}} \leq 2^{-m} \quad \text { for all } n .
\end{gathered}
$$

Put $h_{n}=\sum_{m} g_{n}^{m} \in X$. Since $f \wedge n_{m} 1=\sum_{k=1}^{m-1} g^{k}$ it follows from (3) and (4) that $f=\left[h_{n}\right] .\left(h_{n}\right)$ is equi-integrable in $X$ because for all $n$

$$
\sum_{k=1}^{m} g_{n}^{k} \leq n_{m} 1, \quad\left\|\sum_{k=m+1}^{\infty} g_{n}^{k}\right\|_{X} \leq 2^{-m} .
$$

1.6. Notation. In analyzing equi-integrable sets we need the following 'normdistribution' of $f \in X$ :

$$
d_{f}(t)=\|f\|-\||f| \wedge t 1\| \text { for } t \in \mathbf{R}^{+} .
$$

Since $X$ is order continuous, $d_{f}$ is continuous, decreasing and $\lim _{t \rightarrow \infty} d_{f}(t)=$ 0 . It follows from 1.3 and $1.5(\mathrm{~b})$ that $d_{f}$ for $f \in \widetilde{X}$ has these properties too.

1.7. Proposition. Let $M \subset X$ be bounded.

(a) If $M$ is an equi-integrable set in $X$, then $\left\{d_{f}, f \in M\right\}$ is a compact subset of $C[0, \infty)$ with respect to uniform convergence.

(b) If $f_{n} \in M$ and $f_{n} \rightarrow 0$ in measure, then

$$
d_{f_{n}}(t) \rightarrow 0 \text { for } n \rightarrow \infty \text { for all } t>0 \text {. }
$$

Proof. (a) Since $\left|d_{f}(t)-d_{f}(s)\right| \leq\|f\| \cdot|s-t|$ the set $\left\{d_{f}, f \in M\right\}$ is equicontinuous on $C[0, s]$ for all $s$. Equi-integrability implies that $d_{f}(t) \rightarrow 0$ for $t \rightarrow \infty$, uniformly for $f \in M$. (b) clear.

\section{THE CHARACTERIZATION THEOREM}

Throughout this section, $X$ denotes a Banach lattice with order continuous norm, represented as a function spaces as in (1).

2.1. Definition. $X$ has the subsequence splitting property (SSP) if for every bounded sequence $\left(f_{n}\right) \subset X$ there is a subsequence $\left(n_{k}\right)$ and sequences $\left(g_{k}\right)$, $\left(h_{k}\right)$ with $\left|g_{k}\right| \wedge\left|h_{k}\right|=0$ and $f_{n_{k}}=g_{k}+h_{k}$ such that

(i) $\left(g_{k}\right)$ is equi-integrable in $X$, i.e. $\left\|\left|g_{k}\right|-\left|g_{k}\right| \wedge t 1\right\| \rightarrow 0$ for $t \rightarrow \infty$ uniformly in $k$.

(ii) The $h_{k}$ 's are pairwise disjoint.

The following examples appear in the literature: 
2.2. Examples. (a) $L_{p}(\Omega, \mu), 1 \leq p<\infty$, and more generally Orlicz-function spaces satisfying the $D_{2}$-condition have the SSP. (See $[11,4]$.)

(b) [7] $q$-concave Banach lattices with $q<\infty$ have SSP. Recall that $X$ is $q$-concave if there is a constant $C$ such that for all $f_{1}, \ldots, f_{n} \in X$

$$
\left\|\sum f_{n}\right\| \geq c\left(\sum\left\|f_{n}\right\|^{q}\right)^{1 / q}
$$

(c) [1] Banach lattices with a uniformly monotone norm have SSP. The norm is uniformly monotone, if for ever $\alpha>0$ there is a $\beta>0$ such that for $f, g \in X_{+}$with $\|f\| \geq \alpha$ and $\|g\| \leq 1$ we have $\|f+g\| \geq\|g\|+\beta$.

(d) Purely atomic Banach lattices have SSP. In this case, SSP is just a restatement of the Bessarga-Pełczyński basis selection principle [5, p. 42].

Since each of the assumptions in (a) to (d) implies that $X_{\mathfrak{A}}$ has order continuous norm, it follows from Theorem 4 below that we have SSP in each of these cases, but we also see that the conditions (a) to (d) are far from being necessary. Indeed, only the relatively small band $\widetilde{X}$ of $X_{\mathfrak{a}}$ has to have order continuous norm.

On the other hand, the following counterexamples are known.

(e) $X=c_{0}$ does not have SSP. (Just consider $f_{n}=\sum_{m=1}^{n} e_{m}, e_{m}=\left(\delta_{m, j}\right)_{j}$.)

(f) [7] There are reflexive, $p$-convex Banach lattices such that $X$ does not have SSP but $X^{*}$ has SSP.

In the following characterization theorem, we need a variant of finite representability (compare [9]).

2.4. Definition. We say that $l_{\infty}^{n}$ 's are equi-normably embedded into $X$ if for every $\varepsilon>0$ there are $f_{i}^{n} \in X_{+}, i=1, \ldots, n, n \in \mathbf{N}$ with

(i) $\left\|f_{i}^{n}\right\|=1, f_{i}^{n} \wedge f_{j}^{n}=0$ for $i \neq j$,

(ii) $\left\|\sum_{i=1}^{n} f_{i}\right\| \leq 1+\varepsilon$, i.e. $\left[f_{1}^{n}, \ldots, f_{n}^{n}\right]$ is $(1+\varepsilon)$ isomorphic to $l_{n}^{\infty}$,

(iii) for every $i$, the sequence $\left(d_{f_{i}^{n}}\right)_{n}$ norm converges in $C[0, \infty)$.

2.5. Theorem. For a Banach lattice $X$ the following statements are equivalent:

(1) $X$ has the subsequence splitting property.

(2) $\widetilde{X}$ has order continuous norm (see Definition 1.1).

(3) $c_{0}$ does not embed in $\widetilde{X}$.

(4) $l_{\infty}^{n}$ cannot be embedded equi-normably in $X$.

Proof. We can assume that $c_{0}$ does not imbed into $X$ since this is implied by all of the above conditions.

$(1) \Rightarrow(2)$. Otherwise, there are pairwise disjoint $A^{m} \in \widetilde{\Sigma}, f=\left[f_{n}\right] \in \widetilde{X}_{+}$ and $\delta>0$ such that $\left\|f \chi_{A^{m}}\right\|>\delta$. Choose a sequence $l_{m}$ with

$$
\left\|f \chi_{A^{m}} \wedge l_{m} 1\right\|>\delta \text {. }
$$


For a fixed $k$ we choose $A_{n}^{m} \in \Sigma, n \in \mathbf{N}, m=1, \ldots, k$, with

$$
\chi_{A^{m}}=\left[\chi_{A_{n}^{m}}\right], \quad f \chi_{A^{m}}=\left[f \chi_{A_{n}^{m}}\right] \text { and } A_{n}^{m} \cap A_{n}^{l}=\varnothing
$$

for $m, l \in\{1, \ldots, k\}, m \neq l$, and all $n$. Since $\mathfrak{A}-\lim _{n} \mu\left(A_{n}^{m}\right)=\mu\left(A^{m}\right)$, $\mathfrak{A}-\lim \left\|f \chi_{A_{n}^{m}} \wedge l_{m} 1\right\|>\delta$ there is an index $n_{k}$ such that for $m=1, \ldots, k$,

$$
\mu\left(A_{n_{k}}^{m}\right) \leq 2 \widetilde{\mu}\left(A^{m}\right), \quad\left\|f_{n_{k}} \chi_{A_{n_{k}}^{m}} \wedge l_{m} 1\right\| \geq \delta .
$$

We will show now that $f_{n_{k}}$ has no subsequence that splits. Otherwise, there were a subsequence $F_{j}=f_{n_{k_{j}}}$, an equi-integrable sequence $g_{j} \in X$, and a pairwise disjoint sequence $h_{j}$ with $F_{j}=g_{j}+h_{j}, g_{j} \wedge h_{j}=0$.

For notational convenience put also $B_{j}^{m}=A_{n_{k_{j}}}^{m}$. To obtain a contradiction we choose $\varepsilon>0$ with

$$
\mu(B)<\varepsilon \Rightarrow \sup _{j}\left\|g_{j} \chi_{B}\right\|<\delta / 4 .
$$

Next we choose $m$ such that $\widetilde{\mu}\left(A^{m}\right)<\varepsilon / 2$ and $j>m$ with

$$
\left\|\chi_{\left\{h_{j} \neq 0\right\}}\right\|<\delta / 4 l_{m} .
$$

Since $\mu\left(B_{j}^{m}\right) \leq 2 \widetilde{\mu}\left(A^{m}\right) \leq \varepsilon$ by (6), we obtain from (7) and (8) that

$$
\left\|F_{j} \chi_{B_{i}^{m}} \wedge l_{m} 1\right\| \leq\left\|g_{j} \chi_{B_{j}^{m}}\right\|+\left\|h_{j} \wedge l_{m} 1\right\| \leq \delta / 4+l_{m} \cdot \delta / 4 l_{m}=\delta / 2 .
$$

On the other hand, (6) implies the contradiction $\left\|F_{j} \chi_{B_{j}^{m}} \wedge l_{m} 1\right\| \geq \delta$.

(2) $\Rightarrow(1)$. For a bounded sequence $\left(f_{n}\right) \in X$, we can write $f=\left[f_{n}\right]=g+h$ with $g \in \widetilde{X}, h \in \widetilde{X}^{\perp}$. By Proposition 1.5 there are sequences $\left(g_{n}\right)$ and $\left(h_{n}\right)$ with $g=\left[g_{n}\right], h=\left[h_{n}\right]$ such that $\left(g_{n}\right)$ is equi-integrable in $X$ and $h_{n}$ goes to zero in measure. Hence $\left\|f_{n}-g-h_{n}\right\| \stackrel{\mathfrak{A}}{\rightarrow} 0$ and there is a subsequence $n_{k}$ with $\left\|f_{n_{k}}-g_{n_{k}}-h_{n_{k}}\right\| \stackrel{k}{\rightarrow} 0$.

$(2) \Rightarrow(4)$. Assume to the contrary that there are $\left(f_{i}^{n}\right) \subset X, i=1, \ldots, n$, $n \in \mathbf{N}$, with the following properties

(i) $f_{1}^{n}, \ldots, f_{n}^{n}$ are pairwise disjoint for all $n,\left\|f_{i}^{n}\right\|=1$.

(ii) $f_{1}^{n}, \ldots, f_{n}^{n}$ are $(1+\varepsilon)$-equivalent to the unit vector basis of $l_{\infty}^{n}$.

(iii) $\left(d_{f_{i}^{n}}\right)_{n=i, i+1, \ldots}$ uniformly converges to some $d_{i}$ on $[0, \infty)$.

Put $f_{i}^{n}=0$ for $i>n$. Denote by $g_{i}$ the $\tilde{X}$-component of $f_{i}=\left[f_{i}^{n}, n \in \mathbf{N}\right]$. Since

$$
\begin{aligned}
\left\|g_{i} \wedge m 1\right\|_{\widetilde{X}} & =\left\|f_{i} \wedge m 1\right\|=\mathfrak{A}-\lim _{n}\left\|f_{i}^{n} \wedge m\right\| \\
& =1-\lim _{n} d_{f_{i}^{n}}(m)=1-d_{i}(m)
\end{aligned}
$$

and $\lim _{t \rightarrow \infty} d_{i}(t)=0$ we have $\left\|g_{i}\right\|_{\widetilde{X}}=1$. The $g_{i}$ 's are also pairwise disjoint by (i). We get from (ii) that $f=\left[\sum_{i=1}^{n} f_{i}^{n}\right] \in X_{\mathfrak{x}}$ with $\|f\| \leq 1+\varepsilon$. If $g$ is the $\widetilde{X}$-component of $f$ we have $g_{i} \leq g$ for all $i \in \mathbf{N}$ and the $g_{i}$ 's span $l^{\infty}$ 
in $\tilde{X}$. Therefore, $\widetilde{X}$ cannot be order continuous if $l_{n}^{\infty}$ 's embed equinormably into $X$.

(4) $\Rightarrow(3)$. Assume that $c_{0}$ embeds into $\tilde{X}$, i.e., there is a pairwise disjoint sequence $f_{i} \in \tilde{X},\left\|f_{i}\right\|=1$, which is $(1+\varepsilon)$-equivalent to the unit vector basis of $c_{0}$. For a fixed $k \in \mathbf{N}$ we choose representations $\left(f_{i}^{n}\right)_{n}$ of $f_{i}$ for $i=1, \ldots, k$ with $f_{i}^{n} \wedge f_{j}^{n}=0$ for $i \neq j$ and $n \in \mathbf{N}$. There is a $U_{k} \in \mathfrak{A}$ such that for all $n \in U_{k}$

$$
\left\|\sum_{i=1}^{k} f_{i}^{n}\right\| \leq 1+\varepsilon, \quad\left\|f_{i}^{n}\right\| \geq 1-\varepsilon \text { for } i=1, \ldots, k .
$$

This implies already that $\left[f_{1}^{n}, \ldots, f_{k}^{n}\right]$ is $(1+\varepsilon)$-isomorphic to $l_{\infty}^{k}$ for all $n \in U_{k}$. It follows from Proposition 1.3 and the equi-continuity (compare Proposition 1.7) of the functions $d_{f_{i}^{n}}, n \in \mathbf{N}$, that $\mathfrak{A}-\lim _{n} d_{f_{i}^{n}}=d_{f_{i}}$ with respect to uniform convergence. Therefore we can choose $n_{k} \in U_{k}$ with

$$
\sup _{t>0}\left|d_{f_{i}^{n_{k}}}(t)-d_{f_{i}}(t)\right|<\frac{1}{k} \quad \text { for } i=1, \ldots, k .
$$

$(3) \Rightarrow(2)$. A Banach lattice, which is not order continuous contains $l^{\infty}[14$, Theorem 5.14].

2.6. Corollary. Every rearrangement invariant function space $X$ which does not contain $c_{0}$, has the subsequence splitting property.

Proof. $c_{0}$ is not contained in $X$ iff every increasing sequence $f_{n} \leq f_{n+1} \leq \cdots$ with $\left\|f_{n}\right\| \leq C$ has a sup in $X$ and $f_{n} / \sup f_{n}$ in norm (e.g. [14, Proposition 5.15]). It follows now from Example 1.2.b that if $X$ has this property, $\tilde{X}$ has it too.

There is also a more direct proof of the corollary: By Example 1.2.b, we can assume that $X$ is a rearrangement invariant space on $[0,1]$. For a bounded sequence $f_{n} \in X$ we choose by Helley's theorem a subsequence of $f_{n}^{*}$ (which we call again $f_{n}^{*}$ ) such that $f_{n}^{*} \rightarrow f$ pointwise to some measurable function $f$. By order continuity we have $\left.f\right|_{[1 / m, 1]} \in X$ for all $m$ and $\left\|\left.f\right|_{[1 / m, 1]}\right\| \leq$ $\sup _{n}\left\|f_{n}\right\|$. Hence $f \in X$ by the Fatou property. For every $m$ we choose $n_{m}$ such that $\left\|\left(f_{n_{m}}^{*} \vee f-f\right) \chi_{[1 / m, 1]}\right\| \leq \frac{1}{m}$. Then

$$
g_{m}=f_{n_{m}} \chi_{\left\{\left|f_{n_{m}}\right| \leq f_{n_{m}}^{*}(1 / m)\right\}}
$$

is equi-integrable in $X$ and $h_{m}=f_{n_{m}}-g_{m}$ goes to zero in measure.

2.7. Corollary. $X$ and $X^{*}$ have the subsequence splitting property iff $\tilde{X}$ is reflexive. In this case $\widetilde{X}^{*}=\widetilde{X}^{*}$.

Proof. " $\Rightarrow$ " Since $\tilde{X}$ has order continuous norm, we have by [12, p. 29],

$$
\tilde{X}^{*}=\tilde{X}^{\times}=\left\{f \in L_{1}(\tilde{\Omega}, \tilde{\mu}) \mid \int f \cdot g d \tilde{\mu}<\infty \quad \text { for all } g \in \tilde{X}\right\} .
$$

We have $L_{\infty}(\widetilde{\Omega}, \tilde{\mu}) \subset \widetilde{X}^{*} \subset \widetilde{X}^{\times}$with $\|f\|_{\widetilde{X}^{*}}=\sup \left\{\int f \cdot g d \tilde{\mu}:\|g\|_{\widetilde{X}} \leq 1\right\}$. 
Indeed, since $\widetilde{X^{*}}$ has order continuous norm, we can represent $f \in \widetilde{X^{*}}$ as $f=\left[f_{n}\right]$ with an equi-integrable sequence $f_{n}$ in $X^{*}$ (cf. Proposition 1.5). Choose $g_{n} \in X$ with $\left\|g_{n}\right\|=1$ and $f_{n}\left(g_{n}\right)=\left\|f_{n}\right\|_{X^{*}}$. Put $\left[g_{n}\right]=\bar{g}+\underline{g}$ with $\bar{g} \in \widetilde{X}, \underline{g}=\left[\underline{g}_{n}\right] \in \widetilde{X}^{\perp}$ and note that $\mathfrak{A}$ - $\lim f_{n}\left(\underline{g}_{n}\right)=0$ since $\left(f_{n}\right)$ is equi-integrable in $X^{*}$. Hence

$$
\|f\|_{\widetilde{X}^{*}}=\mathfrak{A}-\lim \left\|f_{n}\right\|=\mathfrak{A}-\lim f_{n}\left(\bar{g}_{n}\right) \leq f(\bar{g}) \leq\|f\|_{\widetilde{X}^{\times}} .
$$

The reverse inequality is clear.

For $f \in \widetilde{X}^{\times}$we have $f \wedge n 1 \in L_{\infty}(\widetilde{\mu}) \subset \widetilde{X^{*}}$ and $\|f \wedge n 1\|_{\widetilde{X}^{*}} \leq\|f\|_{X^{\times}}$. Since $\widetilde{X^{*}}$ does not contain $c_{0}$, it follows from [12] 1.c.4 that $f=\sup _{n} f \wedge n 1 \in \widetilde{X}^{*}$. Hence $\widetilde{X}^{*}=\widetilde{X}^{*}$ has also order continuous norm and it follows from [12] 1.c.5 that $\widetilde{X}$ is reflexive. " $\Leftarrow$ " Use [12] 1.c.5.

Next we characterize the SSP in terms of a 'uniform' order continuity property.

2.8. Theorem. For a Banach lattice $X$, the following are equivalent

(1) $X$ has the subsequence splitting property

(2) $X$ has an equivalent lattice norm $|\|\cdot\||$ such that for every $d \in C(0, \infty)$ with $0<d(t) \rightarrow 0$ for $t \rightarrow \infty$ we have

$$
\sup \left\{\left|\left\|\chi_{A} f\right\|\right|: f \in X \text { with } d_{f} \leq d\right\} \rightarrow 0 \text { for } \mu(A) \rightarrow 0 .
$$

(3) $X$ has an equivalent lattice norm $|\|\cdot\||$ such that a set $M \subset X$ is equi-integrable in $X$ if and only if $\left\{d_{f}: f \in M\right\}$ is a compact subset of $C(0, \infty)$ with the sup-norm.

Remark. The typical renorming we use in (2) and (3) is

$$
|\|f\||=\|f\|+\sum_{k=m}^{\infty} 2^{-k}\|f\|_{k}, \quad\|f\|_{k}=\sup \left\{\left\|\chi_{A} f\right\|: \mu(A) \leq \frac{1}{k}\right\} .
$$

Observe that $X=\left(L_{2}[-1,0] \oplus L_{2}[0,1]\right)_{\infty}$ has SSP but does not satisfy (2) or (3). Indeed, if we choose $f_{n} \in X$ such that the $\left.f_{n}\right|_{(-1,0]}$ are pairwise disjoint with $\left\|\left.f_{n}\right|_{[-1,0]}\right\|=1$ and the $\left.f_{n}\right|_{[0,1]}$ are the Rademacher functions then $d_{f_{n}}(t)=0$ for $t>1$, although $f_{n}$ is not equi-integrable.

Proof. (1) $\Rightarrow(3)$ We observed already in 1.7 that for an equi-integrable set $M$ the set $\left\{d_{f}, f \in M\right\}$ is compact in $C[0, \infty)$. To prove the converse we may restrict ourselves to sets $M$ of positive functions. We use the renorming (9). Let $M \subset(X,|\|\cdot\||)$ be such that $\left\{d_{f}: f \in M\right\}$ is compact in $C[0, \infty)$. For $f_{n} \in M$ with $t \rightarrow\left|\left\|f_{n} \wedge t 1\right\|\right|$ converging uniformly on $[0, \infty)$ to a function $d \in C[0, \infty)$, we have to show that $\left(f_{n}\right)$ is equi-integrable. Otherwise, by (1) there are a subsequence of $f_{n}$ (which we denote again by $f_{n}$ ), an equi-integrable sequence $g_{n}$ and a pairwise disjoint sequence $\left(h_{n}\right)$ with

$$
f_{n}=h_{n}+g_{n}, \quad h_{n} \wedge g_{n}=0, \quad\left\|h_{n}\right\| \geq \delta>0 .
$$


By Proposition 1.7 we can assume that $t \rightarrow\left|\left\|g_{n} \wedge t 1\right\|\right|$ also converges uniformly to $d$. On the other hand, there is an $M<\infty$ and $N \in \mathbf{N}$ such that

$$
\begin{aligned}
& \left\|g_{n}\right\|_{m}=\sup \left\{\left\|\chi_{A} g_{n}\right\|: \mu(A) \leq \frac{1}{m}\right\} \leq \delta / 4 \text { for all } n \text { and } m \geq M, \\
& \left\|h_{n}\right\|_{M} \geq 3 \delta / 4 \quad \text { for all } n \geq N .
\end{aligned}
$$

For $n \geq N$ and all $t \in(0, \infty)$ we obtain

$$
\begin{aligned}
& \left|\left\|f_{n} \wedge t 1\right\|\right| \geq\left\|g_{n} \wedge t\right\|+\sum_{m=1}^{M-1} 2^{-m}\left\|g_{n} \wedge t 1\right\|_{m}+\sum_{m=M}^{\infty} 2^{-m}\left\|h_{n} \wedge t 1\right\|_{m} \\
& \geq\left|\left\|g_{n} \wedge t 1\right\|\right|-\sum_{m=M}^{\infty} 2^{-m}\left\|g_{n}\right\|_{m}+2^{-M}\left(\left\|h_{n}\right\|_{M}-\left\|h_{n}-h_{n} \wedge t 1\right\|_{M}\right) \\
& \geq\left|\left\|g_{n} \wedge t 1\right\|\right|+2^{-M}\left(\delta / 2-\left\|h_{n}-h_{n} \wedge t\right\|\right) .
\end{aligned}
$$

Hence for every $n \geq N$ there is a $t \in(0, \infty)$ with

$$
\left|\left\|f_{n} \wedge t 1\right\|\right|-\left|\left\|g_{n} \wedge t 1\right\|\right| \geq 2^{-M-2} \delta>0 .
$$

But we observed earlier that this difference should go to zero uniformly.

(3) $\Rightarrow(2)$. Choose an equivalent lattice norm on $X$ for which (3) holds and observe that the set $M=\left\{d_{f}: f \in X ; d_{f} \leq d\right\}$ is compact in $C[0, \infty]$ by the same argument we used in the proof of Proposition 1.7.

(2) $\Rightarrow(1)$. Choose an equivalent lattice norm on $X$ for which (2) holds.

Let $\left(f_{n}\right)$ be a bounded, positive sequence in $X$. Since the functions $s \rightarrow$ $\left\|f_{n} \wedge s 1\right\|, n \in \mathbf{N}$, are equi-continuous on every finite interval $[0, t]$, we can choose a subsequence of $f_{n}$ (call it again $\left(f_{n}\right)$ ) such that the functions $s \rightarrow$ $\left\|f_{n} \wedge s 1\right\|, n \in \mathbf{N}$, converge uniformly on every $[0, m], m \in \mathbf{N}$. Put $C=$ $\sup _{t>0} \lim _{n}\left\|f_{n} \wedge t\right\|$.

For every $m \in \mathbf{N}$ we choose $n_{m}$ and $t_{m}$ with $n_{m}>n_{m-1}, t_{m}<t_{m+1} \rightarrow \infty$ such that

$$
C+\frac{1}{m} \geq\left\|f_{n} \wedge t_{m}\right\| \geq C-\frac{1}{m} \text { for all } n \geq n_{m} .
$$

Put now $g_{m}=f_{n_{m}} \wedge t_{m} 1, h_{m}=f_{n_{m}}-f_{n_{m}} \wedge t_{m} 1$ and $d(t)=C-\inf _{m}\left\|g_{m} \wedge t\right\| . d$ is decreasing and assume for a moment that $\operatorname{dim}_{t \rightarrow \infty} d(t)=0$. Since $\left\|g_{m}\right\| \rightarrow C$ by (10) we can assume that $\left\|g_{m}\right\|=C$. Then $d_{g_{m}}(t) \leq d(t)$ for $t \geq 0$ and it follows from (2) that $\left(g_{m}\right)$ is equi-integrable. Observe that $\left\{h_{m} \neq 0\right\} \subset$ $\left\{f_{n_{m}} \geq t_{m}\right\}=A_{m}$ and that $\mu\left(A_{m}\right) \rightarrow 0$ for $m \rightarrow \infty$ since $t_{m} \rightarrow \infty$ and $\left(f_{n}\right)$ is bounded in $L_{1}(\mu)$. Now we obtain the required splitting for $f_{n_{m}}=g_{m}+h_{m}$ by applying a disjointification procedure to $\left(h_{m}\right)$.

It remains to show that $\lim _{t \rightarrow \infty} d(t)=0$. Otherwise there is a $l \in \mathbf{N}$ and a subsequence $\left(m_{k}\right)$ with $\left\|g_{m_{k}} \wedge k\right\| \leq C-2 l^{-1}$.

Since $\left\|g_{m}\right\|=C$ we have $m_{k} \rightarrow \infty$ for $k \rightarrow \infty$. Therefore, for a $k$ with $t_{m_{k}} \wedge k \geq t_{l}$ and $n_{m_{k}} \geq n_{l}$ we get from (10) that

$$
\left\|g_{m_{k}} \wedge k\right\| \geq\left\|f_{n_{m_{k}}} \wedge t_{l}\right\| \geq C-l^{-1} .
$$

This contradiction completes the proof. 


\section{REFERENCES}

1. M. Akcoglu and L. Sucheston, On ergodic theory and truncated limits in Banach lattices, Proc. of the 1983 Oberwolfach Measure Theory Conference, Lecture Notes in Math., vol. 1089, Springer-Verlag, Berlin and New York, 1984, pp. 241-262.

2. K. Chong and N. Rice, Equi-measurable rearrangements of functions, Queen's Papers in Pure and Applied Mathematics, no. 28, Kingston, 1971.

3. D. Dacunha-Castelle and J. L. Krivine; Sous-espace de $L_{1}$, Israel J. Math. 26 (1977), 320-351.

4. D. Dacunha and M. Schreiber, Techniques probabilistes pour l' 'étude de problèmes d' isomorphismes entre espaces de Banach, Ann. Inst. Henri Poincaré 10 (1974), 229-277.

5. J. Diestel, Sequences and series in Banach spaces, Springer-Verlag, Berlin and New York, 1984.

6. P. Dodds and D. Fremlin, Compact operators in Banach lattices, Israel J. Math. 34 (1979), 287-320.

7. T. Figiel, N. Ghoussoub and W. B. Johnson, On the structure of nonweakly compact operators on Banach lattices, Math. Ann. 257 (1981), 317-334.

8. N. Ghoussoub and W. B. Johnson, Factoring operators through Banach lattices not containing $C[0,1]$, Math. Ann. (to appear).

9. S. Heinrich, Ultraproducts in Banach space theory, J. Reine Angew. Math. 313 (1980), 72-104.

10. W. B. Johnson, B. Maurey, G. Schechtman and L. Tzafriri, Symmetric structures in Banach spaces, Mem. Amer. Math. Soc. no. 217, 1979.

11. M. Kadec and A. Pelczyński, Bases, lacunary sequences and complemented subspaces in the spaces $L_{p}$, Studia Math. 21 (1962), 161-176.

12. J. Lindenstrauss and L. Tzafriri, Classical Banach spaces. II, Springer-Verlag, Berlin and New York, 1979.

13. B. de Pagter and A. Schep, Measures of non-compactness of operators in Banach lattices, J. Funct. Anal. (1988), 31-55.

14. H. H. Schaefer, Banach lattices and positive operators, Berlin and New York, 1974

15. L. Weis, On the computation of some quantities in the theory of Fredholm operators, Rend. Circ. Mat. Palermo (2) Suppl. no. 5 (1984), 109-133.

Department of Mathematics, Louisiana State University, Baton Rouge, Louisiana 70803 